\title{
Associations Between Prolonged Intubation and Developing Post-extubation Dysphagia and Aspiration Pneumonia in Non-neurologic Critically Ill Patients
}

\author{
Min Jung Kim, MD, Yun Hee Park, MD, Young Sook Park, MD, You Hong Song, MD \\ Department of Physical Medicine and Rehabilitation, Samsung Changwon Hospital, \\ Sungkyunkwan University School of Medicine, Changwon, Korea
}

Objective To identify the associations between the duration of endotracheal intubation and developing postextubational supraglottic and infraglottic aspiration (PEA) and subsequent aspiration pneumonia.

Methods This was a retrospective observational study from January 2009 to November 2014 of all adult patients who had non-neurologic critical illness, required endotracheal intubation and were referred for videofluoroscopic swallowing study. Demographic information, intensive care unit (ICU) admission diagnosis, severity of critical illness, duration of endotracheal intubation, length of stay in ICU, presence of PEA and severity of dysphagia were reviewed. Results Seventy-four patients were enrolled and their PEA frequency was 59\%. Patients with PEA had significantly longer endotracheal intubation durations than did those without (median [interquartile range]: 15 [9-21] vs. 10 [6-15] days; $\mathrm{p}=0.02$ ). In multivariate logistic regression analysis, the endotracheal intubation duration was significantly associated with PEA (odds ratio, 1.09; 95\% confidence interval [CI], 1.01-1.18; $\mathrm{p}=0.04$ ). Spearman correlation analysis of intubation duration and dysphagia severity showed a positive linear association ( $\mathrm{r}=0.282$, $\mathrm{p}=0.02$ ). The areas under the receiver operating characteristic curves (AUCs) of endotracheal intubation duration for developing PEA and aspiration pneumonia were 0.665 (95\% CI, 0.542-0.788; $\mathrm{p}=0.02$ ) and 0.727 (95\% CI, 0.614$0.840 ; \mathrm{p}=0.001$ ), respectively.

Conclusion In non-neurologic critically ill patients, the duration of endotracheal intubation was independently associated with PEA development. Additionally, the duration was positively correlated with dysphagia severity and may be helpful for identifying patients who require a swallowing evaluation after extubation.

Keywords Intratracheal intubation, Deglutition disorders, Aspiration pneumonia, Critical illness, Fluoroscopy

() This is an open-access article distributed under the terms of the Creative Commons Attribution Non-Commercial License (http://creativecommons.org/ licenses/by-nc/4.0) which permits unrestricted noncommercial use, distribution, and reproduction in any medium, provided the original work is properly cited. Copyright $\odot 2015$ by Korean Academy of Rehabilitation Medicine 


\section{INTRODUCTION}

Although dysphagia is not a disease but rather a symptom of another disease or intervention, it can lead to a variety of complications such as dehydration, malnutrition, aspiration or secretion of food, and death [1]. Aspiration often leads to pneumonia, which is associated with poor outcomes. In patients with dysphagia, risk of aspiration pneumonia is 11 times greater than it is in those without [2].

Post-extubation dysphagia (PED), which is defined as the inability to effectively transfer food and liquid from the mouth into the stomach, has been reported in $3 \%$ to $62 \%$ of adult patients who undergo prolonged mechanical ventilation [1]. Endotracheal tubes can cause direct trauma and impairments in the anatomy and physiology of the pharynx and larynx that may be associated with subsequent swallowing disorders [3-7]. Most importantly, focal ulceration and/or inflammation can damage the vocal cords and cause granulation tissue and scarring. Arytenoid dislocation and subluxation can result in impaired glottic closure during swallowing. Furthermore, impaired swallowing reflex was detected after extubation. Additionally, the recurrent laryngeal nerve can be compressed by the cuff, resulting in vocal cord paralysis.

Clinical studies regarding the association between endotracheal intubation duration and PED reported conflicting results [8-13]. Recently, a number of studies reported associations between the intubation duration and PED or between PED and patient outcomes, but these were limited by inaccurate diagnostic tests for dysphagia, such as bedside swallow evaluation (BSE) and patientreported questionnaires, and inappropriate subjects who had neurologic impairment [14-17]. Videofluoroscopic swallowing study (VFSS) is highly sensitive for and specific to aspiration and is the most commonly utilized instrumental assessment tool for evaluating oropharyngeal dysphagia [18-20]. However, previous studies of PED that used VFSS were limited and insufficient $[9,21,22]$. These studies used VFSS only on patients who failed the BSE, or they enrolled patients with neurologic impairment. Therefore, the aim of this study was to evaluate the associations between endotracheal intubation duration and VFSS-identified PED and between the duration and developing aspiration pneumonia in non-neurologic critically ill patients.

\section{MATERIALS AND METHODS}

Subjects

We retrospectively reviewed the medical records of patients who were referred for VFSS at a teaching university hospital from January 2009 to November 2014. We selected critically ill patients with the following criteria: age $\geq 18$ years, intensive care unit (ICU) admission, mechanical ventilation with endotracheal intubation for any duration, and swallowing difficulty. Patients were excluded if they had a history of neurologic disease that could also cause dysphagia (e.g., stroke, motor neuron disease, high cervical cord injury); had received trauma, surgical intervention or radiation in the head and neck area (including tracheostomy); or did not complete the standard VFSS protocol (e.g., cognitive dysfunction).

\section{VFSS protocol}

Standardized VFSS was performed using a fluoroscopy unit (KXO-BOXM; Toshiba Co., Tokyo, Japan). Swallowing images on the lateral projection were acquired while the subjects were seated. First, $3 \mathrm{~mL}$ of thick liquid was provided to the subjects; the liquid was a $200 \%$ liquid solution that was made from $140 \%$ barium sulfate liquid (Solotop Suspension 140; Taejoon Pharm, Seoul, Korea) with additional barium sulfate powder. Then, $3 \mathrm{~mL}$ of rice porridge and boiled rice, which contained the $140 \%$ barium solution, were provided. Finally, $3 \mathrm{~mL}$ of a thin, diluted $70 \%$ barium solution was provided. Each test was performed twice on each subject. A videofluoroscopytrained radiographer and two experienced clinicians conducted each study.

\section{VFSS analysis}

All VFSS procedures were recorded, and the images were analyzed by a clinician under the supervision of a rehabilitation specialist. VFSS findings of penetration or aspiration, which was defined as the passage of contrast into the airway, above and below the vocal cord respectively, were considered as the presence of post-extubational supraglottic and infraglottic aspiration (PEA) [23]. A functional dysphagia scale (FDS) that used a 100-point scoring system was used to quantify the dysphagia severity [24].

\section{Outcome measure}

In this study, the first analysis was to determine the risk 
factors for the presence of PEA. In this analysis, the primary independent variable of interest was the duration of endotracheal intubation, and other variables of interest included demographic information, ICU admission diagnosis, severity of critical illness and length of stay (LOS) in ICU. Age and sex were considered demographic characteristics. ICU admission diagnosis was categorized as follows: respiratory, cardiac, non-respiratory sepsis, renal, surgical, intoxication and miscellaneous etiology. Simplified acute physiologic score (SAPS) II [25], acute physiologic and chronic health evaluation (APACHE) II [26], and sequential organ failure assessment (SOFA) [27] scores were used to evaluate the severity of the subjects' critical illnesses and were measured from data at ICU admission. These variables were selected based on the study aim and the existing literature [15-17].

Other analyses in this study were to identify the associations between endotracheal intubation duration and dysphagia severity and between the duration and developing aspiration pneumonia. FDS score was acquired from VFSS analysis and used to quantify the dysphagia severity. Based on the literature $[28,29]$, aspiration pneumonia was diagnosed with the following features: the history of suspected aspiration (e.g., choking during a meal), fever with elevated white blood cell counts, abnormal chest radiographs, and no other source of the fever.

All of these data were compiled by reviewing medical records and analyzing VFSS results. All of these protocols were approved by the Institutional Review Board of our hospital.

\section{Statistical analysis}

SPSS program ver. 18.0 for Windows (IBM SPSS Inc., Armonk, NY, USA) was used for statistical analysis, and any p-values less than 0.05 were considered significant. The Kolmogorov-Smirnov test was performed for age, SAPS II score, APACHE II score, SOFA score, LOS in ICU, endotracheal intubation duration and FDS score. These data were not normally distributed, and, consequently, they were reported using median and interquartile range (IQR). They were compared between two groups, which were divided by presence of PEA, using the Mann-Whitney U-test. Categorical variables such as sex, ICU admission diagnosis and number of patients with pneumonia were reported as frequency and proportion and were descriptively analyzed.
The effects of independent variables, including intubation duration, on the presence of PEA were evaluated using logistic regression analysis, with associations presented as odds ratios (OR). First, univariate logistic regression analysis was applied for each individual variable. Then, variables were included in the multivariate logistic regression analysis if they exhibited significant association with the outcomes in univariate analysis $(\mathrm{p}<0.1)$.

Finally, to address the effects of intubation duration on patient outcomes, Spearman correlation analysis and receiver operating characteristic (ROC) curve analysis were performed. The former was used to identify the linear association between duration and FDS score. The latter was used to obtain the optimal duration cut-off values for developing PEA and aspiration pneumonia, which were calculated as Sensitivity+Specificity-1 (maximal Youden's index).

\section{RESULTS}

Between January 2009 and November 2014, a total of 1,136 patients were referred for VFSS. Among them, 74 patients (median age [IQR]: 75 years [71-79]; 46 males) satisfied our criteria and were included in this study. The most common disease category for their admission to ICU was a surgical disease $(n=17,23 \%)$. The median (IQR) scores for disease severity were as follows: SAPS II, 44 (40-54); APACHE II, 21 (18-26); and SOFA, 7 (5-9). The median (IQR) for LOS in ICU and endotracheal intubation duration were 15 (8-29) and 12 (7-18) days, respectively (Table 1). Based on their VFSS results, the patients were divided into two groups: 44 with and 30 without PEA. Patients with PEA had significantly longer intubation durations than did those without (15 [9-21] days vs. 10 [6-15] days; $\mathrm{p}=0.02) ;$ SAPS II scores (42 [38-49] vs. 54 [41-64]; $\mathrm{p}=0.001)$, APACHE II scores (19 [17-23] vs. 25 [19-30]; $\mathrm{p}=0.002)$, and SOFA scores (6 [4-8] vs. 9 [5-11]; $\mathrm{p}=0.02$ ) were significantly higher in those without PEA.

In the univariate logistic regression analysis, the endotracheal intubation duration (OR, 1.09; 95\% confidence interval [CI], 1.02-1.17; $\mathrm{p}=0.02$ ) and all three disease severity scores-SAPS II (OR, 0.93; 95\% CI, 0.89-0.97; $\mathrm{p}=0.001$ ), APACHE II (OR, 0.90; 95\% CI, 0.83-0.98; $\mathrm{p}=0.01$ ) and SOFA (OR, 0.84; 95\% CI, 0.73-0.96; $\mathrm{p}=0.01$ )—exhibited unadjusted associations with PED of $\mathrm{p}<0.1$; these variables were included in the multivariate analysis (Table 2). Based on the multivariate analysis, intubation duration 
Table 1. Patient characteristics and comparison of the patients with and without post-extubational aspiration

\begin{tabular}{|c|c|c|c|c|}
\hline Characteristic & Total $(n=74)$ & Aspiration (-) (n=30) & Aspiration $(+)(n=44)$ & p-value \\
\hline Age (yr) & $75(71-79)$ & $75(65-77)$ & $75(71-80)$ & 0.365 \\
\hline Male & $46(62.0)$ & $16(53.0)$ & $30(68.0)$ & \\
\hline \multicolumn{5}{|l|}{ ICU diagnosis } \\
\hline Respiratory & $13(18.0)$ & $5(17.0)$ & $8(18.0)$ & \\
\hline Cardiac & $14(19.0)$ & $6(20.0)$ & $8(18.0)$ & \\
\hline Sepsis (non-respiratory) & $12(16.0)$ & $4(13.0)$ & $8(18.0)$ & \\
\hline Renal & $4(5.0)$ & $4(13.0)$ & $0(0)$ & \\
\hline Surgical & $17(23.0)$ & $3(10.0)$ & $14(32.0)$ & \\
\hline Intoxication & $8(11.0)$ & $6(20.0)$ & $2(5.0)$ & \\
\hline Other & $6(8.0)$ & $2(7.0)$ & $4(9.0)$ & \\
\hline \multicolumn{5}{|l|}{ Severity of critical illness } \\
\hline SAPS II score & $44(40-54)$ & $54(41-64)$ & $42(38-49)$ & $0.001^{*}$ \\
\hline APACHE II score & $21(18-26)$ & $25(19-30)$ & $19(17-23)$ & $0.002 *$ \\
\hline SOFA score & $7(5-9)$ & $9(5-11)$ & $6(4-8)$ & $0.019 *$ \\
\hline \multicolumn{5}{|l|}{ ICU factors } \\
\hline ICU LOS (day) & $15(8-29)$ & $16(8-27)$ & $15(4-39)$ & 0.877 \\
\hline Duration of intubation (day) & $12(7-18)$ & $10(6-15)$ & $15(9-21)$ & $0.016^{*}$ \\
\hline \multicolumn{5}{|l|}{ Severity of dysphagia } \\
\hline FDS score & $23(12-40)$ & $10(4-14)$ & $35(24-57)$ & $0.000^{*}$ \\
\hline \multicolumn{5}{|l|}{ Pneumonia } \\
\hline Pneumonia affected & $28(38.0)$ & $8(27.0)$ & $20(45.0)$ & \\
\hline
\end{tabular}

Values are presented as median (interquartile range) or number (\%).

ICU, intensive care unit; SAPS, simplified acute physiologic score; APACHE, acute physiologic and chronic health evaluation; SOFA, sequential organ failure assessment; LOS, length of stay; FDS, functional dysphagia scale.

* $\mathrm{p}<0.05$ by Mann-Whitney U-test.

was significantly associated with PEA (OR, 1.09; 95\% CI, 1.01-1.18; $\mathrm{p}=0.04)$.

Spearman correlation analysis of endotracheal intubation duration and FDS score showed a positive linear association $(\mathrm{r}=0.282, \mathrm{p}=0.02)$. In the ROC curve analysis, the areas under the ROC curves (AUCs) for developing PEA and aspiration pneumonia were 0.665 (95\% CI, 0.542-0.788; $\mathrm{p}=0.02$ ) and 0.727 (95\% CI, 0.614-0.840; $\mathrm{p}=0.001)$, respectively. The optimal cut-off values for intubation duration were 12.5 days (sensitivity $63.6 \%$, specificity $73.3 \%$ ) for PEA and 8.5 days (sensitivity $100 \%$, specificity $47.8 \%$ ) for aspiration pneumonia (Fig. 1).

\section{DISCUSSION}

This retrospective single-cohort study of non-neurologic critically ill patients with endotracheal intubation shows that the duration of endotracheal intubation may be associated with developing PEA. In addition, duration had a positive correlation with dysphagia severity and may be associated with an increased risk of developing aspiration pneumonia after extubation. Many studies have used VFSS for dysphagia, but studies of PED were limited and insufficient. In a systematic review of 1,489 studies of PED, only 3 of 14 accepted studies used VFSS for diagnosing PED [1]. However, 2 of these studies used VFSS only on patients who failed BSE [9,22], and the other enrolled patients with neurologic impairment [21]. This study excluded patients with neurologic disorders in order to minimize any effects of underlying disease, enrolled only patients who underwent VFSS, and regarded only patients with VFSS-identified supraglottic and infraglottic aspiration as PED, described as PEA in the text, for strict diagnosing, even though the study was retrospective.

Previous studies regarding the association between endotracheal intubation duration and PED reported con- 
Table 2. Logistic regression analysis of factors associated with post-extubational aspiration

\begin{tabular}{|c|c|c|c|c|}
\hline & \multicolumn{2}{|c|}{ Univariate } & \multicolumn{2}{|c|}{ Multivariate } \\
\hline & OR (95\% CI) & p-value & OR (95\% CI) & p-value \\
\hline \multicolumn{5}{|l|}{ Primary variable } \\
\hline Duration of intubation & $1.09(1.02-1.17)$ & $0.015^{*}$ & $1.09(1.01-1.18)$ & $0.035^{*}$ \\
\hline Age & $1.03(0.99-1.08)$ & 0.176 & & \\
\hline Male & $1.88(0.72-4.89)$ & 0.198 & & \\
\hline \multicolumn{5}{|l|}{ ICU diagnosis } \\
\hline Respiratory & (Reference) & & & \\
\hline Cardiac & $0.83(0.18-3.88)$ & 0.816 & & \\
\hline Sepsis (non-respiratory) & $1.25(0.24-6.44)$ & 0.790 & & \\
\hline Renal & $0.00(0.00-0.00)$ & 0.999 & & \\
\hline Surgical & $2.92(0.55-15.56)$ & 0.210 & & \\
\hline Intoxication & $0.21(0.03-1.47)$ & 0.115 & & \\
\hline Other & $1.25(0.16-9.54)$ & 0.830 & & \\
\hline \multicolumn{5}{|l|}{ Severity of critical illness } \\
\hline SAPS II score & $0.93(0.89-0.97)$ & $0.001^{*}$ & $0.94(0.85-1.03)$ & 0.181 \\
\hline APACHE II score & $0.90(0.83-0.98)$ & $0.011^{*}$ & $1.00(0.87-1.16)$ & 0.980 \\
\hline SOFA score & $0.84(0.73-0.96)$ & $0.013^{*}$ & $0.97(0.77-1.21)$ & 0.756 \\
\hline ICU LOS & $1.02(0.99-1.05)$ & 0.150 & & \\
\hline
\end{tabular}

OR, odds ratio; CI, confidence interval; ICU, intensive care unit; SAPS, simplified acute physiologic score; APACHE, acute physiologic and chronic health evaluation; SOFA, sequential organ failure assessment; LOS, length of stay. ${ }^{*} \mathrm{p}<0.05$ by logistic regression analysis.

(A)

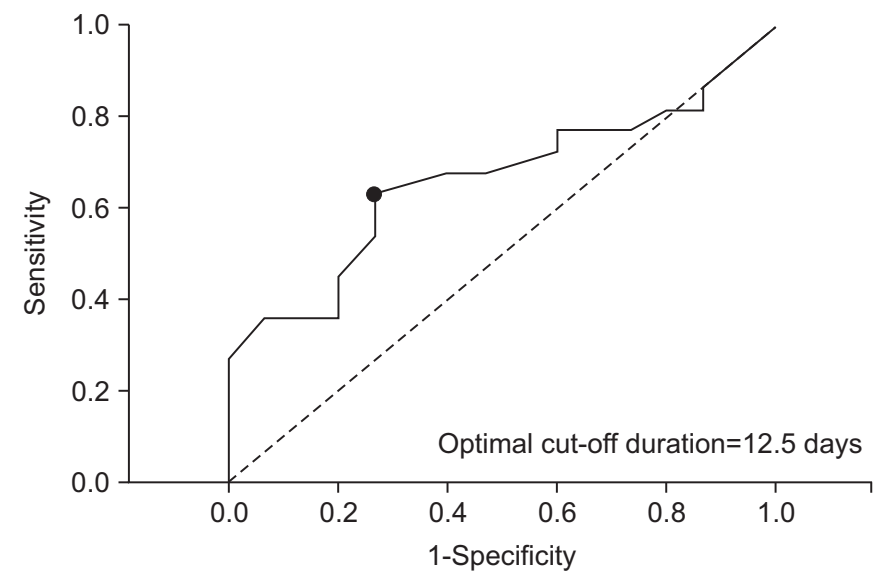

(B)

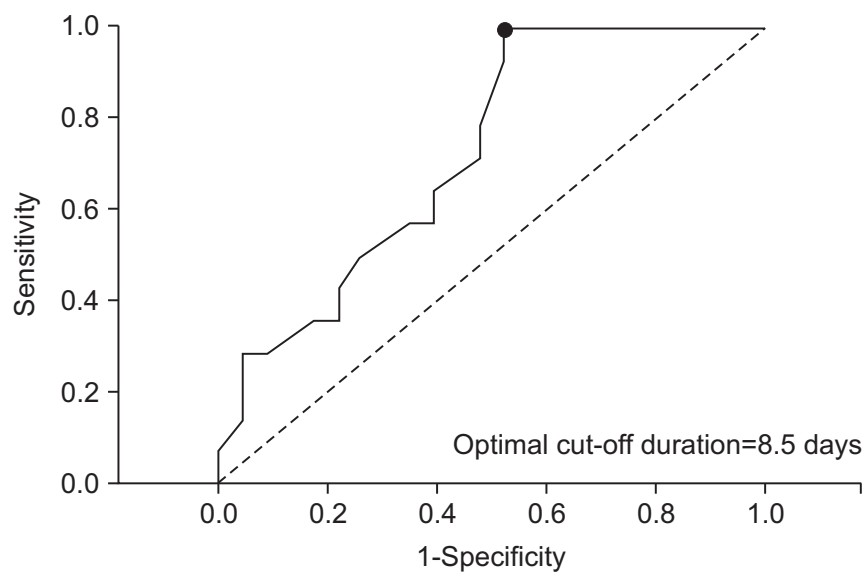

Fig. 1. ROC curves of endotracheal intubation duration for developing post-extubational aspiration (A) and aspiration pneumonia (B) in non-neurologic critically ill patients. The optimal cut-off values (dots on the curves) for duration, which were calculated as Sensitivity + Specificity - 1, were 12.5 days for post-extubational aspiration (AUC, 0.665 ; 95\% confidential interval, $0.542-0.788 ; \mathrm{p}=0.016$; sensitivity $63.6 \%$, specificity $73.3 \%$ ) and 8.5 days for aspiration pneumonia (AUC, $0.727 ; 95 \%$ confidential interval, $0.614-0.840 ; \mathrm{p}=0.001$; sensitivity $100 \%$, specificity $47.8 \%$ ). ROC, receiver operating characteristic; AUC, area under the ROC curve. 
flicting results [8-13], but there are a number of possible explanations for this disagreement: variable evaluations for assessing dysphagia; heterogeneity of subjects; small sample sizes; and statistical analyses that did not adjust for confounding factors. Recently, some studies have used regression analysis to adjust for confounding and showed positive associations. Macht et al. [16] conducted a retrospective, observational cohort study with 2,484 critically ill patients, irrespective of intubation duration, and reported that mechanical ventilation for more than 7 days was independently associated with moderate or severe dysphagia (OR, 2.84; 95\% CI, 1.78-4.56; $\mathrm{p}<0.01$ ) in patients without tracheostomy after adjustment for age, gender and severity of illness. In the present study, although the sample size was small, intubation duration was significantly associated with PEA (OR, 1.09; 95\% CI, 1.01-1.18; $\mathrm{p}=0.04$ ) based on multivariate logistic regression analysis (Table 2). Based on the results, PEA risk is 1.82 times higher 7 days after intubation and continues to increase. Because previous results were acquired from moderate or severe dysphagia, our result may be comparable with these previous results. Rousou et al. [13] retrospectively reviewed the medical records of 838 consecutive cardiac surgical patients and found a positive association of endotracheal intubation duration with dysphagia (OR, 1.01; 95\% CI, 1.00-1.03, p=0.005). However, because of the shortness of intubation time (intraoperative intubation), differences in patient characteristics (patients with cardiac surgery) and influential intervention for dysphagia (transesophageal echocardiography), it may be difficult to directly compare our data with this result.

The association between intubation duration and dysphagia severity is supported by some previous studies. Barker et al. [9] retrospectively compared intubation duration between patients with and without PED, and duration in the former group was roughly 1.6 times longer than in the latter group $(142.4 \pm 63.0$ hours vs. $87.1 \pm 43.3$ hours; $\mathrm{p}<0.001$ ). In this study, longer postoperative endotracheal intubation showed significant association with delayed return to normal oral feeding on time-related analysis using the Kaplan-Meier method $(\mathrm{p}<0.001)$. Macht et al. [16] divided the severity of dysphagia into three degrees (none, mild and moderate or severe) and analyzed the incremental tendency of endotracheal intubation duration along with increased PED severity using univariate comparison with the Kruskal-
Wallis test (median [IQR] for duration: 4 [2-7] days without dysphagia vs. 6 [3-11] days with mild dysphagia vs. 10 [5-17] days with moderate or severe dysphagia; $\mathrm{p}<0.01$ ). In the present study, endotracheal intubation duration showed positive correlation with dysphagia severity, measured as FDS score, by Spearman correlation analysis $(\mathrm{r}=0.282, \mathrm{p}=0.02)$. All of these studies found the association between intubation duration and dysphagia severity in separate ways. However, the results were not entirely valuable because of their retrospective designs. Additional research with prospective designs is necessary to clearly demonstrate the association.

Only a few studies have examined the associations between patient outcomes and PED. Both El Solh et al. [11] and Ajemian et al. [8] reported that neither PEA pneumonia nor any deaths were found in their studies. In these studies, diet modification intervened in the association between swallowing evaluations and outcome measures, and thus preventive actions should be considered. Macht et al. [16] reported that PED was associated with poor outcomes including pneumonia, reintubation and death. In this study, the moderate or severe PED group contained more patients who had been affected by pneumonia than did the none or mild groups ( $3 \%$ vs. $6 \%$ vs. $14 \%$; $\mathrm{p}<0.01$ ). In the present study, we evaluated the effect of endotracheal intubation duration-rather than dysphagia severity-on patient outcomes. Although the definition of aspiration pneumonia remains vague and unclear [30], intubation duration showed moderate predictive power for developing the illness (AUC, 0.727; 95\% CI, 0.614-0.840; $\mathrm{p}=0.001$ ) (Fig. 1). In addition, the optimal cut-off value obtained from the maximal Youden's index was calculated as 8.5 days with $100 \%$ sensitivity and $47.8 \%$ specificity. In consideration of its sensitivity, this result may be helpful for identifying patients who require a swallowing evaluation after extubation.

In this study, the patients without PEA showed higher critical illness severity than did those with PEA (median SAPS II score: 54 vs. $42, \mathrm{p}=0.001$; median APACHE II score: 25 vs. $19, \mathrm{p}=0.002$; median SOFA score: 9 vs. 6 , $\mathrm{p}=0.019$ ) (Table 1). Apart from damage to the endotracheal tube, a number of other mechanisms could possibly cause PED [19]. Systemic inflammatory response syndrome, sepsis and multi-organ failure are important risk factors for ICU-acquired weakness and/or critical illness neuromyopathy $[31,32]$, which are potential PED triggers. 
Indeed, in patients with prolonged mechanical ventilation, neuromuscular recovery is typically prolonged and incomplete [33]. All of the patients who were enrolled in this study were referred for VFSS, likely because dysphagia was suspicious. Although the association between critical illness severity and PEA development was excluded by multivariate logistic regression analysis, dysphagia influenced by more severe critical illness and early resolution of dysphagia attributable to recovery from critical illness and early extubation could be possible explanations for this result.

In the present study, we used VFSS to assess dysphagia severity as well as standard diagnosis. VFSS is the most commonly used instrumental study for evaluating oropharyngeal dysphagia $[18,20]$. Moreover, it was the most widely available diagnostic test for PED in a national survey in the United States (98\% of all respondents), followed by fiberoptic endoscopic evaluation of the swallow (FEES, 41\%) [34]. However, the survey reported that the most common diagnostic test to evaluate for PED was a BSE, which was not a gold standard because of its poor sensitivity as well as poor interjudge and intrajudge reliability $[35,36]$. Another multisite prospective study of extubated patients with acute lung injury reported that the prevalence of post-extubation swallowing assessment was only $44 \%$ and that BSE (58 patients) was used more frequently than VFSS (6 patients) [37]. Importantly, aspiration on VFSS has been associated with significantly increased risk of developing subsequent pneumonia [38]. In addition, in their systematic review of PED, Skoretz et al. [1] proposed that using sensitive swallow assessments on all enrollees would best represent the characteristics of dysphagia following extubation. Although VFSS and FEES, which are major tests for dysphagia assessment, had controversies regarding their respective strengths and limitations $[39,40]$, active utilization of instrumental assessments for PED may be helpful for reducing the development of aspiration pneumonia and subsequent mortality.

There were a number of potential limitations in our study. First, because of the study's retrospective nature, it was not possible to estimate the incidence of PEA. In previous studies, dysphagia frequencies following endotracheal intubation varied, ranging from $3 \%$ to $62 \%$ [1]. PEA frequency in our study (59\%) was relatively higher than it was previous results because: 1) VFSS, which is highly sensitive for detecting dysphagia, was used; and 2) VFSSreferred patients who were suspicious for dysphagia were enrolled. In addition, the lack of medical records led to insufficient covariates in the logistic regression analysis. Third, to avoid small sample size, we were not able to include a homogeneous patient group. Instead, we utilized patients' underlying critical illnesses as a variable of regression analysis to rule out their potential association with PEA and aspiration pneumonia. Finally, there are no standard criteria for diagnosing aspiration pneumonia. Although we referred to the previous literature and blindly reviewed medical records, additional prospective studies with more clear definitions should be performed to identify the association between endotracheal intubation duration and developing aspiration pneumonia.

In non-neurologic critically ill patients, the intubation duration was independently associated with developing PEA. Additionally, duration was positively correlated with dysphagia severity, and it may be helpful for identifying patients who require a swallowing evaluation after extubation. Understanding the mechanisms that contribute to PED and developing methods to further address this disorder might decrease morbidity in these critically ill patients and advance critical care.

\section{CONFLICTS OF INTEREST}

No potential conflict of interest relevant to this article was reported.

\section{REFERENCES}

1. Skoretz SA, Flowers HL, Martino R. The incidence of dysphagia following endotracheal intubation: a systematic review. Chest 2010;137:665-73.

2. Martino R, Foley N, Bhogal S, Diamant N, Speechley M, Teasell R. Dysphagia after stroke: incidence, diagnosis, and pulmonary complications. Stroke 2005; 36:2756-63.

3. Stauffer JL, Olson DE, Petty TL. Complications and consequences of endotracheal intubation and tracheotomy: a prospective study of 150 critically ill adult patients. Am J Med 1981;70:65-76.

4. Colice GL, Stukel TA, Dain B. Laryngeal complications of prolonged intubation. Chest 1989;96:877-84.

5. Sue RD, Susanto I. Long-term complications of artifi- 
cial airways. Clin Chest Med 2003;24:457-71.

6. de Larminat V, Montravers P, Dureuil B, Desmonts JM. Alteration in swallowing reflex after extubation in intensive care unit patients. Crit Care Med 1995;23:48690.

7. Colton House J, Noordzij JP, Murgia B, Langmore S. Laryngeal injury from prolonged intubation: a prospective analysis of contributing factors. Laryngoscope 2011;121:596-600.

8. Ajemian MS, Nirmul GB, Anderson MT, Zirlen DM, Kwasnik EM. Routine fiberoptic endoscopic evaluation of swallowing following prolonged intubation: implications for management. Arch Surg 2001;136:434-7.

9. Barker J, Martino R, Reichardt B, Hickey EJ, RalphEdwards A. Incidence and impact of dysphagia in patients receiving prolonged endotracheal intubation after cardiac surgery. Can J Surg 2009;52:119-24.

10. Barquist E, Brown M, Cohn S, Lundy D, Jackowski J. Postextubation fiberoptic endoscopic evaluation of swallowing after prolonged endotracheal intubation: a randomized, prospective trial. Crit Care Med 2001;29:1710-3.

11. El Solh A, Okada M, Bhat A, Pietrantoni C. Swallowing disorders post orotracheal intubation in the elderly. Intensive Care Med 2003;29:1451-5.

12. Hogue CW Jr, Lappas GD, Creswell LL, Ferguson TB Jr, Sample M, Pugh D, et al. Swallowing dysfunction after cardiac operations. Associated adverse outcomes and risk factors including intraoperative transesophageal echocardiography. J Thorac Cardiovasc Surg 1995;110:517-22.

13. Rousou JA, Tighe DA, Garb JL, Krasner H, Engelman RM, Flack JE 3rd, et al. Risk of dysphagia after transesophageal echocardiography during cardiac operations. Ann Thorac Surg 2000;69:486-90.

14. Altman KW, Yu GP, Schaefer SD. Consequence of dysphagia in the hospitalized patient: impact on prognosis and hospital resources. Arch Otolaryngol Head Neck Surg 2010;136:784-9.

15. Macht M, King CJ, Wimbish T, Clark BJ, Benson AB, Burnham EL, Williams A, Moss M. Post-extubation dysphagia is associated with longer hospitalization in survivors of critical illness with neurologic impairment. Crit Care 2013;17:R119.

16. Macht M, Wimbish T, Clark BJ, Benson AB, Burnham EL, Williams A, et al. Postextubation dysphagia is per- sistent and associated with poor outcomes in survivors of critical illness. Crit Care 2011;15:R231.

17. Brodsky MB, Gellar JE, Dinglas VD, Colantuoni E, Mendez-Tellez PA, Shanholtz C, et al. Duration of oral endotracheal intubation is associated with dysphagia symptoms in acute lung injury patients. J Crit Care 2014;29:574-9.

18. Marik PE, Kaplan D. Aspiration pneumonia and dysphagia in the elderly. Chest 2003;124:328-36.

19. Macht M, Wimbish T, Bodine C, Moss M. ICU-acquired swallowing disorders. Crit Care Med 2013;41:2396405.

20. Martin-Harris B, Jones B. The videofluorographic swallowing study. Phys Med Rehabil Clin N Am 2008;19:769-85.

21. Ferraris VA, Ferraris SP, Moritz DM, Welch S. Oropharyngeal dysphagia after cardiac operations. Ann Thorac Surg 2001;71:1792-6.

22. Keeling WB, Lewis V, Blazick E, Maxey TS, Garrett JR, Sommers KE. Routine evaluation for aspiration after thoracotomy for pulmonary resection. Ann Thorac Surg 2007;83:193-6.

23. Rosenbek JC, Robbins JA, Roecker EB, Coyle JL, Wood JL. A penetration-aspiration scale. Dysphagia 1996;11:93-8.

24. Han TR, Paik NJ, Park JW. Quantifying swallowing function after stroke: a functional dysphagia scale based on videofluoroscopic studies. Arch Phys Med Rehabil 2001;82:677-82.

25. Le Gall JR, Lemeshow S, Saulnier F. A new Simplified Acute Physiology Score (SAPS II) based on a European/North American multicenter study. JAMA 1993; 270:2957-63.

26. Knaus WA, Draper EA, Wagner DP, Zimmerman JE. APACHE II: a severity of disease classification system. Crit Care Med 1985;13:818-29.

27. Vincent JL, Moreno R, Takala J, Willatts S, De Mendona A, Bruining H, et al. The SOFA (Sepsis-related Organ Failure Assessment) score to describe organ dysfunction/ failure. On behalf of the Working Group on SepsisRelated Problems of the European Society of Intensive Care Medicine. Intensive Care Med 1996;22:707-10.

28. Lin CW, Chang YC, Chen WS, Chang K, Chang HY, Wang TG. Prolonged swallowing time in dysphagic Parkinsonism patients with aspiration pneumonia. Arch Phys Med Rehabil 2012;93:2080-4. 
29. Seo WH, Oh JH, Nam YH, Sung IY. Clinical study of aspiration pneumonia in stroke patients. J Korean Acad Rehabil Med 1994;18:52-8.

30. Komiya K, Ishii H, Kadota J. Healthcare-associated pneumonia and aspiration pneumonia. Aging Dis 2014;6:27-37.

31. Druschky A, Herkert M, Radespiel-Troger M, Druschky K, Hund E, Becker CM, et al. Critical illness polyneuropathy: clinical findings and cell culture assay of neurotoxicity assessed by a prospective study. Intensive Care Med 2001;27:686-93.

32. De Jonghe B, Sharshar T, Lefaucheur JP, Authier FJ, Durand-Zaleski I, Boussarsar M, et al. Paresis acquired in the intensive care unit: a prospective multicenter study. JAMA 2002;288:2859-67.

33. Kress JP, Hall JB. ICU-acquired weakness and recovery from critical illness. N Engl J Med 2014;370:1626-35.

34. Macht M, Wimbish T, Clark BJ, Benson AB, Burnham EL, Williams A, et al. Diagnosis and treatment of postextubation dysphagia: results from a national survey. J Crit Care 2012;27:578-86.

35. McCullough GH, Wertz RT, Rosenbek JC, Mills RH,
Ross KB, Ashford JR. Inter- and intrajudge reliability of a clinical examination of swallowing in adults. Dysphagia 2000;15:58-67.

36. Ramsey DJ, Smithard DG, Kalra L. Early assessments of dysphagia and aspiration risk in acute stroke patients. Stroke 2003;34:1252-7.

37. Brodsky MB, Gonzalez-Fernandez M, Mendez-Tellez PA, Shanholtz C, Palmer JB, Needham DM. Factors associated with swallowing assessment after oral endotracheal intubation and mechanical ventilation for acute lung injury. Ann Am Thorac Soc 2014;11:154552.

38. Pikus L, Levine MS, Yang YX, Rubesin SE, Katzka DA, Laufer I, et al. Videofluoroscopic studies of swallowing dysfunction and the relative risk of pneumonia. AJR Am J Roentgenol 2003;180:1613-6.

39. Langmore SE. Evaluation of oropharyngeal dysphagia: which diagnostic tool is superior? Curr Opin Otolaryngol Head Neck Surg 2003;11:485-9.

40. Aviv JE. Prospective, randomized outcome study of endoscopy versus modified barium swallow in patients with dysphagia. Laryngoscope 2000;110:563-74. 